\title{
Vernakalant: Perception of state of health in patients with a recent-onset atrial fibrillation
}

\author{
Diego Conde ${ }^{1}$, Juan Pablo Costabel ${ }^{1}$, Martin Aragon ${ }^{1}$, \\ Florencia Lambardi ${ }^{1}$, Marcelo Trivi ${ }^{2}$ \\ ${ }^{1}$ Cardiovascular Emergency Care Section, Instituto Cardiovascular de Buenos Aires, Argentina \\ ${ }^{2}$ Clinical Cardiology Service, Instituto Cardiovascular de Buenos Aires, Argentina
}

\begin{abstract}
Background: Vernakalant is a new, safe and effective drug used intravenously, which has proved to be more rapid in converting recent onset atrial fibrillation $(A F)$ to sinus rhythm compared to placebo, amiodarone, propafenone, and flecainide in clinical studies. Until now no study has been conducted comparing the perception of state of health in patients who received vernakalant versus propafenone or flecainide for conversion of recent-onset $A F$. The aim of our study is to compare the change of perception of state of health from screening to hour 2 in patients treated with vernakalant and propafenone or flecainide for conversion of recent-onset $A F$.

Methods: Eighty hemodynamically stable patients with recent onset AF without structural heart disease were prospectively included. A single oral dose of propafenone $600 \mathrm{mg}$ was administered to 30 patients, 30 patients received intravenous vernakalant and the remaining 20 patients received a single oral dose of flecainide $300 \mathrm{mg}$. Clinical, laboratory variables and perception of state of health from screening to hour 2 treated with these drugs measured by the EQ-5 D quality-of-life assessments visual analog scale were recorded.

Results: Baseline characteristics were similar in the three groups. Treatment with vernakalant resulted in a significantly greater improvement in patient perception of state of health at hour 2 compared with propafenone and flecainide. In the vernakalant group, a mean increase (from baseline) of 12.1 points was seen compared with a mean increase of 5.4 points in the propafenone group or 5.2 points in flecainide group $(p<0.01)$.

Conclusions: The change of perception of state of health from screening to hour 2 treated with vernakalant had a significantly statistical improvement compared with propafenone or flecainide for conversion recent-onset AF. (Cardiol J 2014; 21, 3: 304-308)
\end{abstract}

Key words: atrial fibrillation, propafenone, flecainide, vernakalant, perception of state of health

\section{Introduction}

Recent-onset atrial fibrillation (AF) is a frequent cause for presentation to the emergency department $[1,2]$. Conversion of recent onset $\mathrm{AF}$ to sinus rhythm with antiarrhythmic drugs reduces the risk of hemodynamic instability, hospitalizations and atrial remodeling seen with persistent $\mathrm{AF}[3,4]$.

Boriani et al. [5] compared oral loading dose of propafenone $600 \mathrm{mg}$ with intravenous propafenone and placebo. At $8 \mathrm{~h}$ either intravenous or oral pro-

Address for correspondence: Diego Conde, MD, Cardiovascular Emergency Care Section, Instituto Cardiovascular de Buenos Aires, Blanco Encalada 1543, Postal Code: 1428, Buenos Aires, Argentina, tel: 5491163816339, fax: 541147877533, e-mail: drconde@hotmail.com

Received: 14.06.2013 Accepted: 20.06.2013 
pafenone were effective in almost two thirds of the patients with a statistical difference versus placebo.

Khan [6] showed that a single oral dose of flecainide $300 \mathrm{mg}$ had a similar time to conversion of $\mathrm{AF}$ to sinus rhythm to intravenous drugs class IC.

This is the reason why an oral loading dose of propafenone $600 \mathrm{mg}$ or a single dose of flecainide $300 \mathrm{mg}$ are used in our center as in other places around the world for conversion of recent onset $\mathrm{AF}$ in patients without structural heart disease.

Vernakalant is a new, safe and effective drug used intravenously for conversion $\mathrm{AF}$ and studied in patients with and without structural heart disease; even after cardiovascular surgery [7-10].

Vernakalant has proved to be more rapid in converting recent onset $\mathrm{AF}$ to sinus rhythm compared to placebo, amiodarone, propafenone, and flecainide $[7,9,11,12]$. Until now no study has been conducted comparing the change of perception of health state before and after in patients who received this new drug versus oral dose of propafenone or flecainide.

The aim of our study is to compare the change of perception of state of health from screening to hour 2 in patients treated with vernakalant and propafenone or flecainide for conversion of recent-onset AF.

\section{Methods}

This is a sequential study in which patients were included in three periods, one for each drug.

From 1 July to 30 October 2012, 30 patients were prospectively and consecutively included, hemodynamically stable with symptomatic recent onset AF (lasting less than $48 \mathrm{~h}$ ) without structural heart disease underwent pharmacological cardioversion and received an initial intravenous dose of vernakalant, $3.0 \mathrm{mg} / \mathrm{kg}$ over $10 \mathrm{~min}$, followed by a 15 min observation period and, if conversion to sinus rhythm did not occur, a second $10 \mathrm{~min}$ infusion of vernakalant at a dose of $2 \mathrm{mg} / \mathrm{kg}$ was administered.

From 1 November 2012 to 31 January 2013, 20 patients were prospectively and consecutively included and received a single oral dose of flecainide $300 \mathrm{mg}$.

From 1 February to 30 April 2013, 30 patients were prospectively and consecutively included and received an oral loading dose of propafenone $600 \mathrm{mg}$.

All patients received the pharmacological cardioversion. If patients persisted with AF after pharmacological cardioversion, the electrical cardioversion was done at $2 \mathrm{~h}$ after intravenous vernakalant or at $8 \mathrm{~h}$ after oral dose of propafenone or flecainide.

Inclusion criteria: Patients $>18$ years old, with AF lasting less than $48 \mathrm{~h}$ and documented by electrocardiogram, weight between $45 \mathrm{~kg}$ and $136 \mathrm{~kg}$, systolic blood pressure $>90 \mathrm{~mm} \mathrm{Hg}$ and $<160 \mathrm{~mm} \mathrm{Hg}$ and diastolic blood pressure $<95 \mathrm{~mm} \mathrm{Hg}$.

Exclusion criteria: Pregnancy, atrial flutter, sinus node disease, QRS duration longer than $140 \mathrm{~ms}$ in non-paced beats, QT interval $>440 \mathrm{~ms}$, heart failure or acute coronary syndrome.

Clinical, laboratory and electrocardiographic variables were recorded. All the patients had continuous electrocardiographic monitoring. Color Doppler echocardiography with measurement of structural and functional parameters were performed to all the patients.

Primary outcome measure: The change of perception of state of health from screening to hour 2 in patients treated with vernakalant and propafenone or flecainide for conversion of recent-onset AF.

Adverse events: Death, sustained hypotension (systolic blood pressure $\leq 90 \mathrm{~mm} \mathrm{Hg}$ ), bradycardia $<40 \mathrm{bpm}$, QT interval $>440 \mathrm{~ms}$, ventricular arrhythmia ( $\geq$ triplets), or any other event that required or prolonged hospitalization were considered adverse events. Other events not meeting the criteria of seriousness, taste disorders, cough, nausea or dizziness were not considered serious adverse events.

The patients will receive anticoagulation therapy after discharge according the recommendation of CHA2DS2-VASc score, but without antiarrhythmics drugs.

Follow-up: Follow-up visits were scheduled 7 days after the index event to evaluate presence of symptoms and maintenance of sinus rhythm.

\section{Statistical analysis}

All calculations were performed using Statistix 8.0 software package. Continuous variables were expressed as median with the corresponding interquartile range (IQR) (p25-p75) and were compared using the Mann-Whitney test. Rates were expressed as percentages and were compared using the $\chi^{2}$ test with Fisher's correction, if applicable. Time taken for conversion to sinus rhythm was illustrated on a graph using the Kaplan-Meier method. The change of perception of state of health from screening to hour 2 was measured by the EQ-5D quality-of-life assessments visual analog scale, which has the Argentine valuation [13]. This inve- 
Table 1. Baseline characteristics.

\begin{tabular}{lccc}
\hline Variable & Propafenone & Vernakalant & Flecainide \\
\hline Male gender & $60 \%$ & $60 \%$ & $70 \%$ \\
Age [years] & $62(54-66)$ & $67(56-69.5)$ & $66(55-69)$ \\
Body mass index $\left[\mathrm{kg} / \mathrm{m}^{2}\right]$ & $26(24-29)$ & $27(25-29.2)$ & $26(23-28)$ \\
Systolic blood pressure $[\mathrm{mm} \mathrm{Hg}]$ & $130(120-142)$ & $127(121.5-130)$ & $129(119-135)$ \\
Diastolic blood pressure $[\mathrm{mm} \mathrm{Hg}]$ & $72(67.7-81.5)$ & $75(69-80)$ & $73(67-75)$ \\
Cardiovascular risk factors: & & & $30 \%$ \\
Diabetes & $20 \%$ & $20 \%$ & $50 \%$ \\
Hypertension & $40 \%$ & $30 \%$ & $70 \%$ \\
Current or former smokers & $60 \%$ & $50 \%$ & $70 \%$ \\
$\quad$ Dyslipidemia & $60 \%$ & $50 \%$ & $20 \%$ \\
Thyroid disorders & $10 \%$ & $10 \%$ & $163(149-162)$ \\
Rate ventricular response per min & $154(147-161)$ & $161(152-166)$ & \\
\hline
\end{tabular}

Table 2. History of atrial fibrillation (AF) and medication.

\begin{tabular}{lccc}
\hline Variable & Propafenone & Vernakalant & \multicolumn{2}{c}{ Flecainide } \\
\hline Previous AF & $10 \%$ & $20 \%$ & $10 \%$ \\
Previous AF ablation & $10 \%$ & $10 \%$ & $20 \%$ \\
Previous treatment: & & & $20 \%$ \\
Beta-blockers & $20 \%$ & $30 \%$ & $0 \%$ \\
Calcium channel blockers & $0 \%$ & $10 \%$ & $0 \%$ \\
Propafenone/Flecainide & $5 \%$ & $0 \%$ & $0 \%$ \\
Amiodarone & $0 \%$ & $10 \%$ & $10 \%$ \\
Anticoagulation & $0 \%$ & $10 \%$ & $40 \%$ \\
CHA2DS2-VASc score: & & & $40 \%$ \\
0 & $30 \%$ & $20 \%$ & $20 \%$ \\
1 & $40 \%$ & $40 \%$ & $0 \%$ \\
2 & $10 \%$ & $30 \%$ & $0 \%$ \\
4 & $20 \%$ & $10 \%$ & $0 \%$ \\
\hline
\end{tabular}

stigation was in accordance with the Declaration of Helsinki. The protocol was reviewed and approved by an institutional review board or ethics committee at Instituto Cardiovascular de Buenos Aires, and written informed consent was obtained from the patients.

\section{Results}

Eighty patients were included; median age was 63 years (54-69.5) and $67.5 \%$ were men.

No significant differences were found between the baseline characteristics and previous events of $\mathrm{AF}$, invasive procedures and medication in the three groups (Tables 1, 2).
Time to conversion to sinus rhythm was $11 \mathrm{~min}$ in the vernakalant group vs. $159 \mathrm{~min}$ (IQR 125-325) in the propafenone group, and $167 \mathrm{~min}$ (IQR 130-315) in flecainide group $(\mathrm{p}<0.01)$ (Fig. 1).

Conversion rate was of $83 \%$ in the propafenone group, $80 \%$ in the flecainide group at $8 \mathrm{~h}$, and $91 \%$ in the vernakalant group at $2 \mathrm{~h}$; yet, this difference was not statistically significant at $8 \mathrm{~h}$ $(\mathrm{p}=$ NS) (Fig. 1).

The stay length in emergency care section was 246 min (IQR 190-280) in the vernakalant group vs. $420 \mathrm{~min}$ (IQR 342-741) in the propafenone group, and $400 \mathrm{~min}$ (IQR 334-725) in flecainide group $(\mathrm{p}<0.01)$ (Fig. 2). 


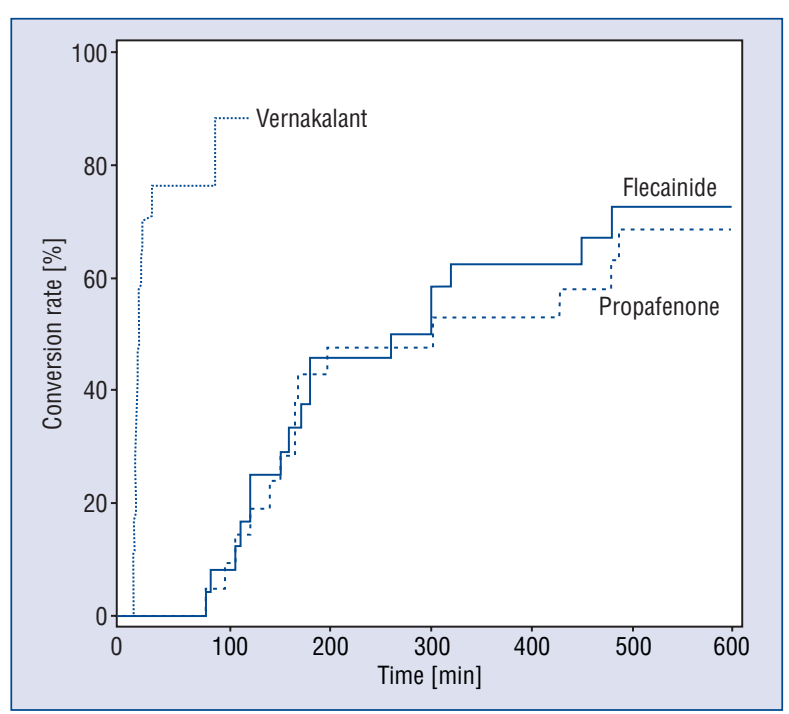

Figure 1. Time to conversion of atrial fibrillation to sinus rhythm.

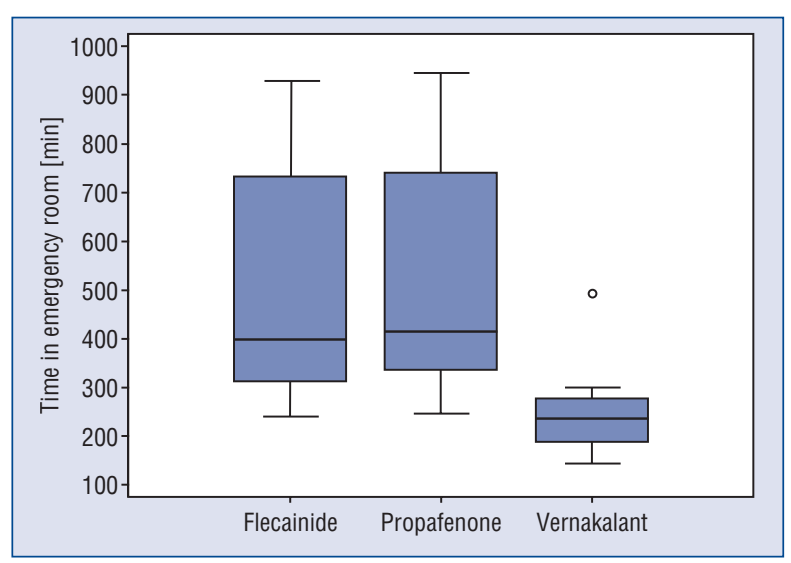

Figure 2. Length of stay in an emergency care section.

Treatment with vernakalant resulted in a significantly greater improvement in patients' perception of state of health (as measured by the EQ-5D quality-of-life assessment visual analog scale) at hour 2 compared with propafenone and flecainide. In the vernakalant group, a mean increase (from baseline) of 12.1 points was seen compared with a mean increase of 5.4 points in the propafenone group or 5.2 points in flecainide group $(\mathrm{p}<0.01)$.

No adverse events were reported.

After 1-week follow-up, all patients who had reverted remained in sinus rhythm. No late adverse events were reported.

\section{Discussion}

Several studies have demonstrated the efficacy of oral propafenone for conversion of recent onset AF to sinus rhythm [5]. Other studies showed that oral flecainide has a similar time to conversion to intravenous propafenone and flecainide [6].

Vernakalant is a novel, relatively atrial-selective antiarrhythmic agent that is used intravenously, prolongs the atrial refractory period but has little effect on ventricular repolarization. It is a multi-ion channel blocker blocking early-activating potassium channels combined with concentration-, voltage- and frequency-dependent blockade of sodium channels [7].

Vernakalant has a rapid distribution and onset of action with a mean half-life elimination of $3 \mathrm{~h}$. Plasma concentrations decline approximately $50 \%$ in $10 \mathrm{~min}$. Restoration of sinus rhythm occurs within $90 \mathrm{~min}$ in $50 \%$ of cases [7-10].

Vernakalant produced a rapid conversion according to the results of the CRAFT study [7] (vs. placebo) or AVRO study [9] (vs. amiodarone). There are two studies which showed that vernakalant is faster in conversion of recent-onset $\mathrm{AF}$ than propafenone and flecainide [11, 12].

There are no other studies designed to compare the change of state of health in patients who were treated with vernakalant vs. propafenone and flecainide.

Our study is the first clinical investigation which analyzed the change of perception of state of health as a primary outcome and showed that vernakalant had a significantly statistical improvement compared with propafenone and flecainide group.

The only study which compared the change of perception of state of health with vernaklant as a secondary outcome was the AVRO study [7], which compared vernakalant vs. amiodarone. In this trial the change of perception of state of health was better in vernaklant group compared with amiodarone group and the results of our study are similar to this one [7].

We suppose that there is a very strong relationship between the short time to conversion of AF to sinus rhythm and the better change of perception of state of health in vernakalant group.

We believe that we need new clinical randomized trials with more patients in this population to be able to reach the certainty that these results are able to extrapolate to the daily practice. 


\section{Limitations of the study}

Not to be a randomized trial is the most important limitation of this study. The sample size may underestimate the differences between the groups. A larger sample size could produce statistically significant differences in the time to conversion and hospital stay length in the vernakalant group.

\section{Conclusions}

The change of perception of state of health from screening to hour 2 treated with vernakalant had a significantly statistical improvement compared with propafenone or flecainide for conversion recent-onset $\mathrm{AF}$.

\section{Conflict of interest: none declared}

\section{References}

1. Go AS, Hylek EM, Phillips KA et al. Prevalence of diagnosed atrial fibrillation in adults: national implications for rhythm management and stroke prevention: The Anticoagulation and Risk Factors in Atrial Fibrillation (ATRIA) Study. JAMA, 2001; 285: 2370-2375.

2. Friberg J, Buch P, Scharling H, Gadsbphioll N, Jensen GB. Rising rates of hospital admissions for atrial fibrillation. Epidemiology, 2003; 14: 666-672.

3. Li H, Easley A, Barrington W, Windle J. Evaluation and management of atrial fibrillation in the emergency department. Emerg Med Clin North Am, 1998; 16: 389-403.
4. Zimetbaum P. Antiarrhythmic drug therapy for atrial fibrillation. Circulation, 2012; 125: 381-389.

5. Boriani G, Capucci A, Lenzi T, Sanguinetti M, Magnani B. Propafenone for conversion of recent-onset atrial fibrillation. A controlled comparison between oral loading dose and intravenous administration. Chest, 1995; 108: 355-358.

6. Khan IA. Single oral loading dose of propafenone for pharmacological cardioversion of recent-onset atrial fibrillation. J Am Coll Cardiol, 2001; 37: 542-547.

7. Roy D, Rowe BH, Stiell IG et al.; CRAFT Investigators. A randomized, controlled trial of RSD1235, a novel anti-arrhythmic agent, in the treatment of recent onset atrial fibrillation. J Am Coll Cardiol, 2004; 44: 2355-2361.

8. Roy D, Pratt CM, Torp-Pedersen C et al.; Atrial Arrhythmia Conversion Trial Investigators. Vernakalant hydrochloride for rapid conversion of atrial fibrillation: A phase 3 , randomized, placebo-controlled trial. Circulation, 2008; 117: 1518-1525.

9. Camm AJ, Capucci A, Hohnloser SH et al.; AVRO Investigators. A randomized active-controlled study comparing the efficacy and safety of vernakalant to amiodarone in recent-onset atrial fibrillation. J Am Coll Cardiol, 2011; 57: 313-321.

10. Kowey PR, Dorian P, Mitchell LB et al.; Atrial Arrhythmia Conversion Trial Investigators. Vernakalant hydrochloride for rapid conversion of atrial fibrillation after cardiac surgery: A randomized, double blind, placebo-controlled trial. Circ Arrhythm Electrophysiol, 2009; 2: 652-659.

11. Conde D, Pablo CJ, Martin A et al. Propafenone versus vernakalant for conversion of recent-onset atrial fibrillation. Cardiovasc Ther, 2013; 31: 377-380.

12. Conde D, Costabel JP, Caro M et al. Flecainide versus vernakalant for conversion of recent-onset atrial fibrillation. Int J Cardiol, 2013; 168: 2423-2425.

13. Augustovski FA, Irazola VE, Velazquez AP, Gibbons L, Craig BM. Argentine valuation of the EQ-5D health states. Value Health, 2009; 12: 587-596. 